\title{
Maximal Exercise-Corrected QT as a Predictor of Coronary Artery Disease: Comparison of Simpler Heart Rate Corrections
}

\author{
Aydın Akyuz, MD, Seref Alpsoy, MD, Dursun Cayan Akkoyun, MD, Hasan Degirmenci, MD, and Niyazi Guler, MD \\ Department of Cardiology, Namık Kemal University Medical School, Tekirdag, Turkey
}

\begin{abstract}
Background and Objectives: The relationship between QT prolongation and myocardial ischemia is widely known. Due to the limited value of ST depression, we aimed to evaluate, by using four simpler heart rate corrections (Bazett, Framingham, Fridericia and Hodges), the value of maximal exercise-QTc prolongation in the diagnosis of coronary artery disease (CAD) presence and severity.
\end{abstract}

Subjects and Methods: We enrolled 234 subjects (mean age 57.3 \pm 9 years, 143 men) who had undergone exercise testing and coronary angiography due to a suspicion of CAD in the study. Evaluating CAD severity with Gensini scoring, the CAD group $(n=122)$ and controls with non-CAD were compared in terms of corrected QT duration at maximal exercise.

Results: Age, gender, hypertension, dyslipidemia, smoking, exercise duration, resting, and peak heart rate were similar between the two groups (all p>0.05). The CAD group had higher raw OT values than the controls $\{268$ (169-438) vs. 240 (168-348), $p<0.001\}$. Although Framingham QTc of $\geq 350 \mathrm{~ms}$ and Fridericia QTc of $\geq 340$ ms were seen to be useful for the diagnosis of CAD, there was no additive diagnostic value of exercise QTc in addition to ST depression. Maximal exercise-0Tc Bazett $(r=0.163, p=0.01)$, Framingham $(r=0.239, p=0.001)$, and Fridericia $(r=0.206, p=0.001)$ equations were weakly positively correlated with Gensini scoring.

Conclusion: The patients with CAD have longer QTc intervals at peak heart rates during exercise. This finding provides insufficient evidence to support routine incorporation of QTC at peak heart rates into exercise test interpretation. (Korean Circ J 2013;43:655-663)

KEY WORDS: Coronary artery disease; Corrected OT; Exercise test.

\section{Introduction}

Both exercise testing and corrected OT (QTc) have been used for detecting heart disease in routine clinical practice. The diagnostic value of $\mathrm{QT}$ prolongation at rest in predicting cardiovascular disease and mortality has been well established. ${ }^{1)}$ Normally, both QT and RR

Received: June 20, 2013

Revision Received: July 19, 2013

Accepted: August 29, 2013

Correspondence: Aydın Akyuz, MD, Department of Cardiology, Namık Kemal University Medical School, Hurriyet Mahallesi Sehit Gökmen Yavuz Caddesi 2/1 kat: 4 Daire: 11 Tekirdag, Turkey

Tel: 90-5424116550, Fax: 90-2822626810

E-mail:ayakyuzq5@gmail.com

- The authors have no financial conflicts of interest.

This is an Open Access article distributed under the terms of the Creative Commons Attribution Non-Commercial License (http://creativecommons. org/licenses/by-nc/3.0) which permits unrestricted non-commercial use, distribution, and reproduction in any medium, provided the original work is properly cited. intervals shorten with exercise due to parasympathetic withdrawal and sympathetic activation. ${ }^{2 / 3)}$ In particular, during the period from rest to exercise, the relationship between QT and RR intervals is complex, due to the fact that QT fails to shorten while the heart rate increases. QT-RR dynamics are affected by age, ischemia, exercise, sex, diabetes mellitus (DM), smoking, and antiarrhytmics drugs, as well as genotypic differences concerning the structure and function of repolarization channels. ${ }^{45)}$

Many formulae, such as logarithmic (Ashman), hyperbolic, linear (Framingham, Hodges, Rautaharju PM), log-linear, and non-linear (Bazett, Fridericia) functions, have been proposed to correct for the heart rate effects on $\mathrm{QT}$ interval. ${ }^{6-10)}$ Because most of them tend to be imprecise in detecting the exact $\mathrm{QT}$ interval at differing heart rates, the linear regression function, which uses rate correction factors, has been recently proposed. ${ }^{10)}$

Although ST-segment depression is the most commonly used criterion for the detection of coronary artery disease (CAD), its sensitivity and specificity during exercise testing is about $65 \%$ and $84 \%$, respectively." Thus, to obtain better electrocardiogram (ECG) analy- 
sis during exercise, some investigators focused on OT changes and, consequently, an increased QT dispersion of $\geq 70$ ms during the exercise has been proposed as a predictor of CAD. ${ }^{12) 13)}$ Although the association of both CAD and increased QTc is a well-known synergistic risk factor of sudden cardiac death, ${ }^{14)}$ no study thus far has investigated the QTc response in terms of simpler heart rate correction formulae to maximal exercise to predict CAD. Thus, we aimed to assess the QTc interval by using the formulae of Bazett, Fridericia, Framingham, and Hodges at maximal exercise to predict the presence and severity of CAD.

\section{Subjects and Methods}

\section{Study participants}

The study prospectively investigated 234 consecutive subjects (mean age $57.3 \pm 9$ years; 91 women aged $56.8 \pm 9$ years, range 36 73 years; 143 men aged $58 \pm 9.5$ years, range $34-74$ years) who required exercise stress testing due to a suspicion of CAD. The first group, the CAD group, was composed of 122 CAD patients with an abnormal exercise test (48 female and 74 male, mean age 55.5 \pm 9.1 years). The second group, the control group, was composed of 112 non-CAD subjects with an abnormal exercise test (43 female and 69 male, mean age $54.9 \pm 8.6$. All subjects had a normal resting 12 lead normal ECG and no history of cardiovascular disease. Throughout the study, the subjects were not allowed to take medication known to affect cardiac repolarisation, such as beta-blockers, calcium antagonist, or other antiarrhytmics. The exclusion criteria for both groups were as follows: atrial fibrillation or flutter, previous myocardial infarction, congestive heart failure, chronic obstructive or other pulmonary diseases, any systemic disease, poor neuromuscular capability, and moderate to severe valvular disease. The study was approved by the local ethics committee. All participants provided informed consent.

\section{Exercise test and QT analysis}

The Bruce protocol was used for the treadmill exercise testing. $85 \%$ of the age-predicted heart rate was considered the main target heart rate. Exercise was continued until the occurrence of ST depression, angina pectoris, dyspnea, and palpitations or exhaustion. During the study, 12-lead ECGs were digitally recorded continuously at $500 \mathrm{~Hz}$ using the CardioSoft exercise ECG system, which has onscreen computer-driven calipers (version 4.14, GE Healthcare, Freiburg, Germany). At least $1 \mathrm{~mm}$ or higher horizontal or downsloping ST-segment depression $80 \mathrm{~ms}$ after the J point was considered abnormal. All stored ECG results, using the standard 12-lead ECG tracing at $50 \mathrm{~mm} / \mathrm{s}$ paper speed at $10 \mathrm{~mm} / \mathrm{mV}$ amplitude, were analyzed offline.
Measurements were taken by two cardiologists. To determine the raw $\mathrm{OT}$ interval, the lead DII and V 5 of each ECG was used because its large-amplitude T wave permitted was sufficiently reliable to determine the end of the T wave as described previously. ${ }^{15)}$ We used a mean value of leads DII and V 5 derived from at least 3 cardiac cycles (heartbeats) and measured from the beginning of the earliest onset of the QRS complex to the end of the T wave. When the $T$ and $U$ waves were close together, we identified the end of the T wave as the point at which its descending limb returns to the TP baseline. When the end of the T waves did not cross the isoelectric line, we determined the end of the T wave by the slope method, wherein it is defined by the intersection point between the tangent drawn at the maximum downslope of the T wave and the isoelectric line (Fig. 1). We did not include cases that had a biphasic T wave or those in which we could not determine an isoelectric line between the $T$ and $P$ wave at the maximal exercise ECG due to ECG artefacts.

The corrected QT was calculated using the following formulae; Bazett's formula $\mathrm{QT} c=\mathrm{QT} / \mathrm{RR}^{1 / 2}$, Framingham $\mathrm{QT} \mathrm{C}=\mathrm{QT}+0.154$ (1-RR), Fridericia formula $\mathrm{QT} \mathrm{C}=\mathrm{QT} / \mathrm{RR}^{1 / 3}$, and Hodges $\mathrm{QT} \mathrm{c}=\mathrm{QT}+0.175$ \{hazard ratio (HR)-60\}, respectively. ${ }^{6-9)}$

\section{Angiographic assessment}

Selective coronary angiography (Integris, Philips Medical Systems, Eindhoven, the Netherlands) was carried out using the Judkins technique through femoral artery access within a maximum of 90 days from the exercise test. The angiographic characteristics were obtained from multiple views for both the left and right coronary arteries. The coronary artery was considered diseased if stenosis in a major epicardial coronary artery was $\geq 50 \%$ in diameter. The Gensini scoring system was used to calculate the severity of CAD. ${ }^{16)}$ The degree of coronary artery stenosis was evaluated according to the consensus opinion of three experienced interventional cardiologists.

\section{Statistical analysis}

All data were analyzed using the Statistical Package for the Social Sciences (SPSS) version 17 (SPSS Inc., Chicago, IL, USA) software package. Data were expressed as mean \pm standard deviation and me-

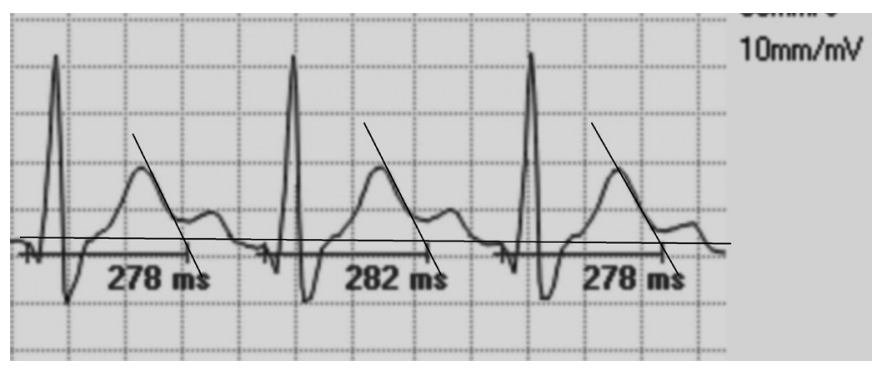

Fig. 1. Illustrates the representative example of exercise raw $0 T$ measurement at $50 \mathrm{~mm} / \mathrm{s}$ paper speed. 
dian (minimum-maximum) values for normal and abnormal distribution, respectively. Categorical data such as gender, diabetes, hypertension, and smoking were denoted as count and percentages, and were compared using the chi-square test. Following a checking of the variables using the Kolmogorov-Smirnov normality test, the mean differences for continuous variables between the two groups were examined by the unpaired Student t-test and the Mann-Whitney $U$ test. After the categorization of OTc values, a chi-square test was performed to compare categorical variables such as gender, hypertension, smoking, diabetes, and the number of coronary artery involvement. Additionally, a paired t-test and Wilcoxon signed rank nonparametric test for paired data were used to compare the differences between the two study groups' pretest and maximal exercise OTc values. Receiver operating characteristic (ROC) curve analysis was performed to identify the optimal cut-off value of the QTC at maximal exercise for the prediction of CAD. A Pearson correlation analysis was used to investigate whether there was a correlation between various OTc formulae and CAD presence and severity. Univariate and multivariate linear regression analysis was used to predict the variables affecting exercise OTc prolongation. A p of less than 0.05 was accepted as statistically significant.

\section{Reliability}

Intraobserver and interobserver variability of OT measurements were assessed by averaging three consecutive OT intervals in lead DII and $V 5$ from 30 randomly selected ECGs. After the measurer was masked to the previous results, the same ECGs were remeasured in random order. The reliability of intraobserver variability was tested using the paired t-test. For interobserver comparisons, a second investigator was blinded to the results of the first investigator. The reliability of interobserver variability was tested using the Student t-test.

\section{Results}

Demographic, clinical characteristics, and exercise electrocardiographic findings of the two groups are shown in Table 1 and 2 . There was no difference between the two groups in terms of age, gender, body mass index (BMI), DM, smoking, and hypertension. ST depression of $\geq 1 \mathrm{~mm}\{n=95(77.8 \%)$ vs. $n=38$ (33.9\%), $p<0.001\}$, Gensini scoring $\{25(10-128)$ vs. $2(0-4), p<0.001\}$, triglyceride (TG) $\{170$ (64-440) vs. 124 (58-330), $p=0.008\}$ and uric acid $\{5.2(1.1-8.3)$ vs. 4.7 (2.4-9.4), $p=0.009\}$ were higher, and high density lipoproteincholesterol (HDL-C) $\{42$ (26-70) vs. 47 (25-87), $p<0.001\}$ was lower in the CAD group than in the control group. There were no significant differences with respect to fasting glucose, haemoglobin, serum creatinine, low density lipoprotein-cholesterol, or exercise test METs (metabolic equivalents) values (all p>0.05) (Table 1).

Resting HR, raw QT interval, OTc formulae results during maximal exercise, systolic, and diastolic blood pressure (BP) in the pretest ex-

Table 1. Baseline characteristics, biochemical results, and Gensini scoring for both groups

\begin{tabular}{|c|c|c|c|}
\hline Variables & CAD group $(n=122)$ & Control group $(n=112)$ & $\mathbf{p}^{*}$ \\
\hline Age (years) & $55.5 \pm 9.1$ & $54.9 \pm 8.6$ & 0.776 \\
\hline Male, n (\%) & $74(60.6)$ & $69(61.6)$ & 0.144 \\
\hline BMI $\left(\mathrm{kg} / \mathrm{m}^{2}\right)$ & $29(23-41)$ & $29(19-45)$ & 0.235 \\
\hline DM, n (\%) & $28(22.9)$ & $24(21.4)$ & 0.441 \\
\hline HT, n (\%) & $38(31.1)$ & $34(30.3)$ & 0.138 \\
\hline LDL-C (mg/dL) & $137 \pm 41$ & $135 \pm 41$ & 0.477 \\
\hline HDL-C (mg/dL) & $42(26-70)$ & $47(25-87)$ & $<0.001$ \\
\hline TG (mg/dL) & $170(64-440)$ & $124(58-330)$ & 0.008 \\
\hline Uric acid (mg/dL) & $5.2(1.1-8.3)$ & $4.7(2.4-9.4)$ & 0.009 \\
\hline METs (unit) & $8.7 \pm 2.3$ & $8.8 \pm 2.4$ & 0.569 \\
\hline ST depression $\geq 1$ mm, n (\%) & 95 (77.8) & $38(33.9)$ & $<0.001$ \\
\hline Gensini score & $25(10-128)$ & $2(0-4)$ & $<0.001$ \\
\hline
\end{tabular}

Data are presented as mean \pm SD, median (minimum-maximum) values and number/percentage. ${ }^{*}$ Chi-square, Mann-Whitney $U$ test and unpaired Student's t-tests. BMI: body mass index, CAD: coronary artery diseases, DM: diabetes mellitus, HT: hypertension, LDL-C: low density lipoprotein-cholesterol, METs: peak metabolic equivalents of exercise test, TG: triglyceride, HDL-C: high density lipoprotein-cholesterol 
Table 2. Resting and maximal exercise testing findings for both groups

\begin{tabular}{|c|c|c|c|}
\hline Variables & CAD group $(n=122)$ & Control group $(n=112)$ & $\mathbf{p}^{*}$ \\
\hline Resting heart rate (bpm) & $81(56-101)$ & $85(50-102)$ & 0.118 \\
\hline Resting OT (ms) & $339 \pm 34$ & $332 \pm 36$ & 0.101 \\
\hline Bazett & $390(311-587)$ & $393(295-488)$ & 0.865 \\
\hline Framingham & $378 \pm 25^{+}$ & $374 \pm 27^{\ddagger}$ & 0.373 \\
\hline Resting systolic BP (mm Hg) & $121 \pm 15$ & $118 \pm 17$ & 0.158 \\
\hline Resting diastolic BP (mm Hg) & $81 \pm 6$ & $78 \pm 8$ & 0.244 \\
\hline Resting to peak HR time (minute) & $6.9 \pm 2.6$ & $7.1 \pm 2.5$ & 0.376 \\
\hline Peak HR (bpm) & $149 \pm 19$ & $150 \pm 20$ & 0.861 \\
\hline \multicolumn{4}{|l|}{ Max. exercise OTc (ms) } \\
\hline Framingham & $360 \pm 34^{+}$ & $340 \pm 29^{\neq}$ & $<0.001$ \\
\hline Fridericia & $350(235-515)^{+}$ & $331(242-461)^{\ddagger}$ & 0.001 \\
\hline Hodges & $411(331-514)^{+}$ & $409(325-497)^{+}$ & 0.601 \\
\hline Peak systolic BP (mm Hg) & $190 \pm 27$ & $195 \pm 26$ & 0.123 \\
\hline Peak diastolic BP (mm Hg) & $95 \pm 12$ & $98 \pm 11$ & 0.156 \\
\hline
\end{tabular}

${ }^{*}$ Chi-square, Mann-Whitney $U$ test and unpaired Student's t-tests, paired t-test and Wilcoxon rank test, ${ }^{\dagger}$ Between resting and maximal exercise in the CAD group ( $p<0.001),{ }^{\ddagger}$ Between resting and maximal exercise in the control group $(p<0.001)$. CAD: coronary artery disease, bpm: beat per minute, BP: blood pressure, HR: heart rate, ms: millisecond

ercise, and peak $H R$, the mean maximal exercise METs values, achievement time from resting $\mathrm{HR}$ to peak $\mathrm{HR}$, systolic, and diastolic $\mathrm{BP}$ in maximal exercise were similar in the two groups (all $\mathrm{p}>0.05$ ). Maximal exercise raw QT interval was longer in the CAD group than in the control group $\{268$ (169-438) ms vs. 240 (168-348) ms, $p<0.001\}$. Among the maximal exercise OTc derivatives findings, only the Hodges formula provided similar results in the two groups, while the Bazett, Fridericia, and Framingham formulae resulted in higher values in the CAD group than in the control group $\{405$ (290-556) ms vs. 391 (291-537) ms, $p=0.015\}$, \{350 (235-515) ms and 331 (279458) $\mathrm{ms}, \mathrm{p}=0.001\}$ and ( $360 \pm 34 \mathrm{~ms}$ vs. $340 \pm 29 \mathrm{~ms}, \mathrm{p}<0.001)$, respectively. In the paired t-test and Wilcoxon rank test analysis, comparing pre-test and maximal exercise OTc derivates, Bazett formula OTc results were similar for both the CAD $(p=0.109)$ and the control groups ( $p=0.523)$, while the Framingham, Fridericia, and Hodges formulae results were significantly different for the two groups (all $p<$ 0.001) (Table 2).

Maximal exercise-Framingham OTc and Fridericia OTc were evaluated by ROC analysis in predicting CAD presence. The closest values of Framingham $350 \mathrm{~ms}$ and of Fridericia $340 \mathrm{~ms}$ to the best specificity and sensitivity points on the ROC curve were identified ffor Framingham QTc: area under the curve (AUC); 0.628 \pm 0.035 (0.5590.696), $p<001$ \} (Fig. 2) and ffor Fridericia OTc: AUC; $0.620 \pm 0.035$

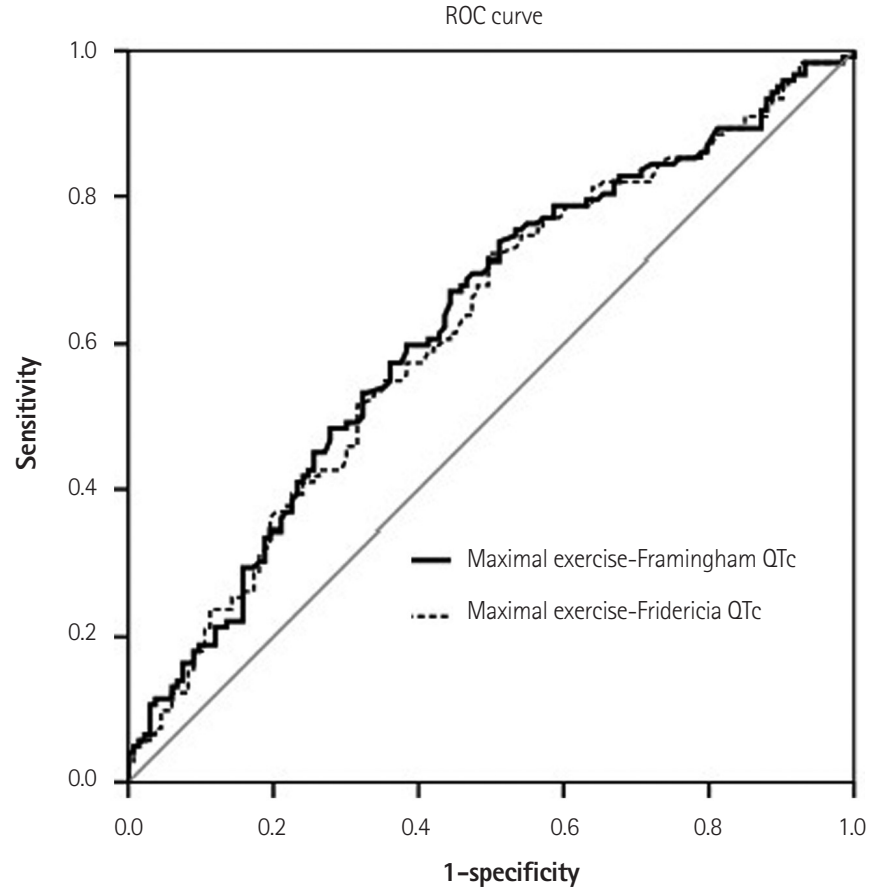

Fig. 2. The sensitivity and specificity of the Framingham and Fridericia formulae in the ROC curve. For the Framingham of $\geq 350 \mathrm{~ms}$, the area under the curve (AUC): $0.628 \pm 0.035$ (0.559-0.696), $p<0.01$ and for the Fridericia OTc: AUC: $0.620 \pm 0.035$ (0.551-0.688), p<0.01. ROC: receiver operating characteristic. 
Table 3. Comparison of the prolongation of Framingham and Fridericia OTc and ST depression between the CAD and the control group

\begin{tabular}{|c|c|c|c|c|c|c|}
\hline & & CAD group & & & ontrol group & \\
\hline & ST depression & No ST depression & Total & ST depression & No ST depression & Total \\
\hline & $n=94(77 \%)$ & $n=28(23 \%)$ & 122 & $n=78(69.6 \%)$ & $n=34(30.4 \%)$ & 112 \\
\hline Framingham OTc $\geq 350 \mathrm{~ms}$ & $54^{*}(44.2 \%)$ & $22(18 \%)$ & $76^{*}(62.2 \%)$ & $22^{*}(19.6 \%)$ & $18(16.1 \%)$ & $40^{*}(35.7 \%)$ \\
\hline Framingham OTc $<350$ ms & $40^{*}(32.7 \%)$ & $6(5 \%)$ & $46^{*}(37.7 \%)$ & $56^{*}(50 \%)$ & $16(14.3 \%)$ & $72^{*}(64.3 \%)$ \\
\hline Fridericia OTc $\geq 340 \mathrm{~ms}$ & $48^{*}(39.3 \%)$ & $18(14.8 \%)$ & $66^{*}(54.1 \%)$ & $29^{*}(25.8 \%)$ & $20(17.9 \%)$ & $49^{*}(43.7 \%)$ \\
\hline Fridericia OTc $<340$ ms & $46(37.7 \%)$ & $10(8.2 \%)$ & $56^{*}(45.9 \%)$ & $49(43.7 \%)$ & $14(12.6 \%)$ & $63^{*}(56.3 \%)$ \\
\hline
\end{tabular}

${ }^{*}$ Chi-square test between the CAD and the control group $(p<0.01)$. CAD: coronary artery disease

(0.551-0.688), $p<001\}$. There was no difference between Framingham the OTC AUC and Fridericia OTC AUC values ( $p=0.846$ ). The sensitivity, specificity, positive and negative predictive values maximal exercise QTc for predicting CAD presence were determined as follows: for Framingham of $\geq 350 \mathrm{~ms} ; 62.2 \%, 64.3 \%, 65.5 \%$, and $61 \%$, and for Fridericia of $\geq 340 \mathrm{~ms} ; 54.1 \%, 56.3 \%, 57.3 \%$, and $55.9 \%$, respectively.

No significant difference was found in terms of ST depression between the two groups. While the number of subjects who had ST depression and peak exercise $\mathrm{QT}$ prolongation in the CAD group was higher than that of the control group ffor Framingham OTc $\geq 350$ ms: $n=54$ (44.2\%) vs. $n=22$ (19.6\%), $p<0.01$ and for Fridericia OTc $\geq 340$ ms: $n=48(39.3 \%)$ vs. $n=29(25.8 \%), p<0.01$, respectively\}, the number of subjects who had no ST depression and peak exercise QT prolongation was similar between the two groups ( $>>0.05$ ) (Table 3). Gender, diabetes, dyslipidemia, hypertension, smoking, and the number of coronary artery vessel involvement were similar in those with peak exercise prolongation and with no peak exercise prolongation (all $p>0.05)$, but no presence of $C A D\{n=39(33.6 \%)$ vs. 46 (38.9\%), $p=0.032\}$ (Table 4). After entered variables of age, BMI, TG, HDL-C, uric acid, METS, peak heart rate, ST depression, and Gensini scoring into univariate and multivariate linear regression anal$y$ sis, age $\{$ unstandardized $\beta=0.86 \pm 0.2, p=0.004$, confidence interval (CI) 95\% from 0.22 to 1.14 and peak heart rate (unstandardized $\beta=-0.58 \pm 0.08, p<0.001, \mathrm{Cl} 95 \%$ : from -0.75 to -0.41 ) were found to be associated with $\mathrm{OT}$ prolongation (Table 5 ).

In the correlation analysis, QTc measurements according to the Bazett $(r=0.163, p=0.01)$, Framingham $(r=0.239, p=0.001)$, and Fridericia $(r=0.206, p=0.001)$ equations were weakly positively correlated with Gensini scoring, but the Hodges equation was not $(r=0.001$, $\mathrm{p}=0.989$ ) (Figs. 3 and 4).

\section{Reliability}

Intra and interobserver reliability comparisons of OT measurements at resting and peak heart rates were similar. For resting QT measurements, the mean differences were 1.1 and $1.3 \mathrm{~ms}, \mathrm{p}=0.417$ and $p=0.296$, respectively. For peak heart rate QT measurements,
Table 4. Comparison of categorical variables according to categorized Framingham QTc $\geq 350$ msn and QTc $<350$ msn groups

\begin{tabular}{lccc}
\hline Variables, $\mathbf{n}(\%)$ & $\begin{array}{c}\text { Framingham } \\
\mathbf{0 T c} \geq \mathbf{3 5 0} \mathbf{~ m s n} \\
\mathbf{n = 1 1 6}(\%)\end{array}$ & $\begin{array}{c}\text { Framingham } \\
\mathbf{0 T c}<\mathbf{3 5 0} \mathbf{~ m s n}\end{array}$ & $\mathbf{p}^{*}$ \\
$\mathbf{n = 1 1 8}(\%)$ & \\
\hline Male gender & $74(63.8)$ & $69(58.5)$ & 0.884 \\
Diabetes & $30(25.8)$ & $22(18.6)$ & 0.546 \\
Dyslipidemia & $41(35.3)$ & $42(35.6)$ & 0.976 \\
Hypertension & $39(33.6)$ & $33(27.9)$ & 0.421 \\
Smoking & $26(22.4)$ & $20(16.9)$ & 0.346 \\
CAD presence & $76(65.5)$ & $46(38.9)$ & 0.032 \\
1-CVI & $25(21.5)$ & $25(21.1)$ & 0.984 \\
2-CVI & $23(19.4)$ & $17(14.4)$ & 0.288 \\
3-CVI & $17(14.6)$ & $15(12.7)$ & 0.156 \\
\hline
\end{tabular}

${ }^{*}$ Chi-square test, coronary artery vessel involvement. CAD: coronary artery disease

the mean differences were 1.2 and $1.4 \mathrm{~ms}, \mathrm{p}=0.398$ and $\mathrm{p}=0.241$, respectively.

\section{Discussion}

We primarily aimed to investigate whether there was OTc prolongation due to ischemia and these formulae were eligible for predicting CAD presence and severity. This study firstly shows that CAD patients have higher peak exercise OTc values. Although the time from resting to peak $\mathrm{HR}$ at maximal exercise was similar in the CAD and non-CAD groups, there was longer raw $Q T$ in the CAD group in the study. Secondly, these findings showed that exercise-induced ischemia was one of the reasons for QT prolongation as well as ST depression, and there was no additive diagnostic value of OTc at peak heart rate in addition to ST depression observed in the study. The percentage of those without ST depression and with longer QTC was similar between the two groups. In addition, exercise QTc sensitivity and specificity were lower than that of ST depression, as described by previous studies, of $68 \%$ and $77 \%$, respectively. ${ }^{17)}$ The sensitivity of ST depression was 77\% in the CAD group. We did not calculate the specificity of ST depression in our study, because our 
Table 5. Univariate and multivariate linear regression analysis for predicting the variables related to exercise QTc prolongation according to the Framingham method

\begin{tabular}{|c|c|c|c|c|}
\hline \multirow{2}{*}{ Variables } & \multirow{2}{*}{$\begin{array}{l}\text { Unstandardized } \\
\text { beta } \pm \text { SE }\end{array}$} & \multirow{2}{*}{ p } & \multicolumn{2}{|c|}{ 95\% confidence interval } \\
\hline & & & Lower bound & Upper bound \\
\hline \multicolumn{5}{|l|}{ Univariate } \\
\hline Age & $0.68 \pm 0.23$ & $<0.001$ & 0.46 & 1.25 \\
\hline BMI & $-0.42 \pm 0.47$ & 0.378 & -1.36 & 0.51 \\
\hline Triglyceride & $2.15 \pm 1.3$ & 0.853 & -0.47 & 0.04 \\
\hline HDL-C & $-0.08 \pm 0.18$ & 0.966 & -0.36 & 0.35 \\
\hline Uric acid & $2.15 \pm 1.3$ & 0.104 & -0.8 & 0.28 \\
\hline METS & $-0.33 \pm 0.89$ & 0.711 & -2.1 & 1.4 \\
\hline Peak heart rate & $-0.54 \pm 0.09$ & $<0.001$ & -0.73 & -0.34 \\
\hline ST depression & $-0.32 \pm 2.7$ & 0.229 & -8.6 & 2.07 \\
\hline Gensini scoring & $0.10 \pm 0.09$ & 0.270 & -0.8 & 0.28 \\
\hline \multicolumn{5}{|l|}{ Multivariate } \\
\hline Age & $0.86 \pm 0.2$ & 0.004 & 0.22 & 1.14 \\
\hline Peak heart rate & $-0.58 \pm 0.08$ & $<0.001$ & -0.75 & -0.41 \\
\hline
\end{tabular}

BMI: body mass index, HDL-C: high density lipoprotein-cholesterol, METs: metabolic equivalents, SE: standard error
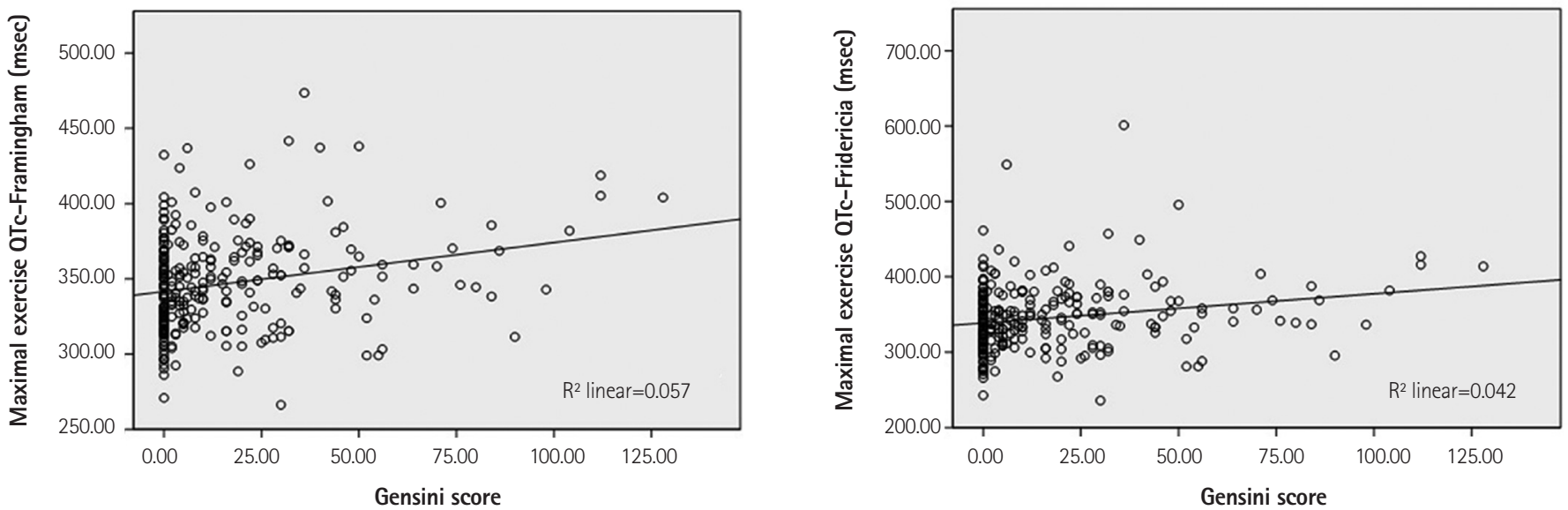

Fig. 3. Shows a positive linear relationship between Gensini scoring and maximal exercise $Q T c$ according to the Framingham $(r=0.239, p=0.001)$ and Fridericia formulae $(r=0.206, p=0.001)$.
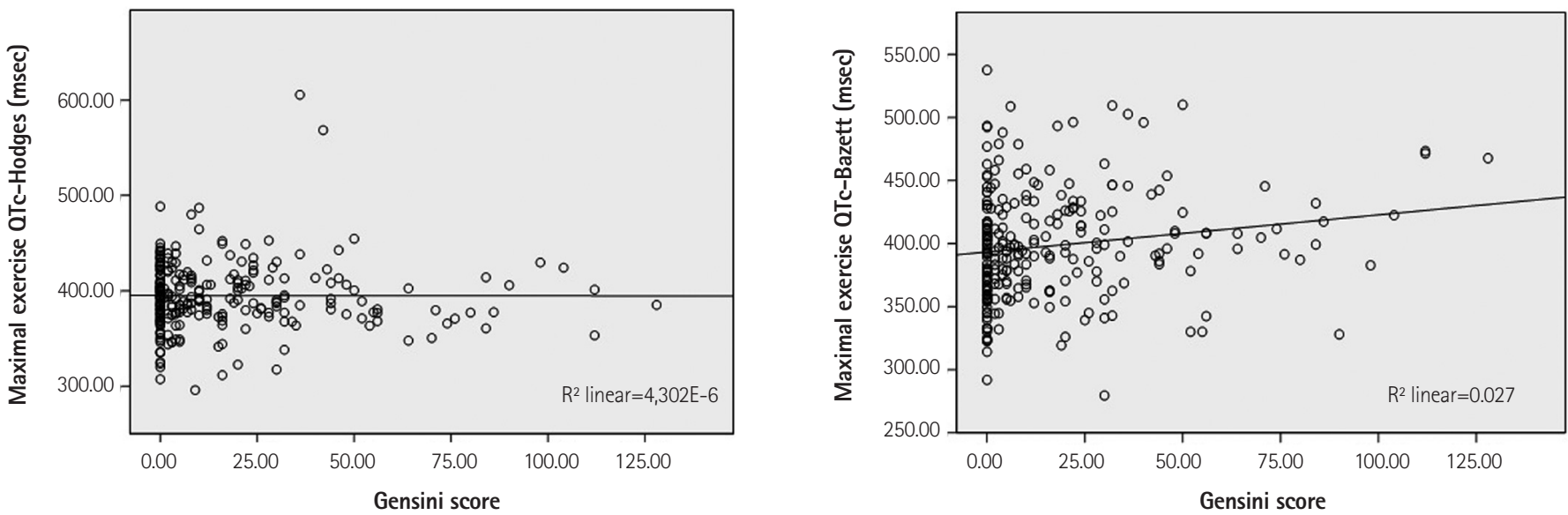

Fig. 4. Gensini scoring is not correlated with maximal exercise QTc according to the Hodges formula $(r=0.001, p=0.989)$, but there is a weakly positive linear relationship between Gensini scoring and maximal exercise OTc according to Bazett $(r=0.163, p=0.01)$. 
control group consisted of non-CAD subjects with ST depression or exercise-induced angina. In other words, their abnormal exercise electrocardiograms were mainly false positives. Finally, we found that age and peak heart rate were found to be related to peak exercise OTc time.

Age, gender, heart rate, underlying rhythm and conduction defects, neuromuscular capability, pulmonary function, physiologic and metabolic state, and or some drugs influence the OT interval. Furthermore, instantaneous ECG at maximal exercise does not estimate the exact OTc interval due to the OT/RR hysteresis. Although numerous methods have been proposed to correct OT intervals for heart rate, Bazett is the most universally adopted method. However, it provides no adequate correction at low, $\leq 60$ beats/min, and high, $\geq 100$ beats/min, heart rates, respectively. The Framingham and Fredericia methods have been proposed for use outside the normal ranges. ${ }^{18)}$ In fact, hysteresis and the time for OT to adapt to RR interval change play an important component of physiology. These methodologies are based on population mean correction factors and do not address intra- or inter-individual variability. Although the $\mathrm{Ba}$ zett, Framingham, Fridericia, and Hodges formulae have not been proposed to determine intra- or inter-individual variability for measuring QTc, especially at peak heart rates, we aimed to evaluate whether there is a difference at peak exercise between CAD patients and non-CAD subjects.

Not surprisingly, we found that these four equations' results were different for both resting and peak exercise heart rates. In this data set, the Framingham and Fridericia formulae led to significantly decreased QTc values, while the Bazett and Hodges correction formulae led to significantly increased OTc values at fast heart rates. Previous studies have shown that Bazett overcorrects the QTc, while Fridericia undercorrects the OTC at fast heart rates. ${ }^{19) 20)}$ Compared to the others at fast heart rates, the distributions of OTc based on Fridericia and Framingham were similar, and showed a significant difference between CAD patients and non-CAD subjects. However, the Hodges QTc revealed no difference. The Bazett QTc at peak heart rates was different between the two groups, but it was not different between the resting and maximal heart rates for the two groups, due to its wider distribution.

The exercise test using ST depression analysis was not more decisive in clinical practice. Making exercise testing more routinely useful related to some OT criteria, such as QT dispersion or T wave analysis, has also been sought in the last decade. ${ }^{16 / 21)}$ In the present study, we compared four different QTc measurement equations and studied their eligibility for the detection of CAD presence and severity. Considered ST depression, our study suggests that Framingham and Fridericia equations were not as eligible as ST depression for detecting $C A D$ presence during peak heart rate during the exer- cise test, and both of them were only weakly correlated with CAD severity. We showed, particularly, that rate-independent OT shortens more in normal controls than in CAD patients at peak exercise. Framingham, a linear method, with OTc $\geq 350$ ms at maximal exercise, was not as satisfying as ST depression in daily routine practice in determining the presence of $C A D$, even though it has moderate sensitivity, 62.2\%, and specificity, 64.3\%, and no additive diagnostic value in addition to ST depression. We found that the Fridericia formula's sensitivity was mildly inferior to that of the Framingham formula in determining CAD presence.

While parasympathetic effects on the heart rate at maximal exercise in healthy subjects are continuous and protective against arrhythmias, ${ }^{22)}$ catecholamine and sympathetic effects of vigorous physical exertion in patients with CAD may trigger myocardial infarction or sudden cardiac death. ${ }^{23)}$ Both graded and sudden intense exercise more commonly trigger cardiac events due to exercise-induced ischemia, resulting in ventricular repolarization heterogeneity, which may be proarrhythmic, by altering the OT rate adaptation. ${ }^{2124) 25)}$ There are many different mechanisms for OTc prolongation in myocardial ischemia, such as tranmural dispersion or repolarization heterogeneity, as well as autonomic neural effects. Normally, exercise-induced ischemia may alter the behaviour of the OT/RR relationship in patients with CAD by decreasing the duration of action potential. ${ }^{26)}$ We considered that the first reason that CAD patients have longer OTc than non-CAD subjects in our study may potentially be related to the longer hysteresis duration for CAD patients. ${ }^{27)}$ Indeed, the substantial intersubject variability of the OT/ $\mathrm{RR}$ relationship has been recently well documented, and the basic QT/RR relationship has been explained by hysteresis, with OT adaptation to instantaneous heart rate changes taking roughly two minutes. ${ }^{28)}$ The precise mechanism of OT hysteresis is not known, however, yet what is understood is that autonomic and non-autonomic factors are responsible. ${ }^{29) 30}$ The second reason may be based on the fact that graded exercise potentially leads to the impairment of cardiac physiological reflexes due to ischemia with greater sympathetic overdrive and acidosis.

Measurement OT intervals at fast heart rates is the first limitation of our study. To minimize misleading results, we particularly excluded cases that appeared to be unreliable for measuring OT interval. We did not use any correction factor for the determinate instantaneous QT/RR relationship for either group, because our study was not designed to characterize the best formula for measuring OTc. This may be seen as a mitigating factor, but we particularly aimed to compare these simpler correction formulae. Second, we assessed OT interval only in lead DII and V 5. OT intervals, in fact, can be measured differently in different leads. Third, OT intervals at peak exercise are influenced both by its antecedent RR interval and by 
the preceding two-minute RR intervals.

In conclusion, the measurement of maximal exercise-OT Framingham QTc of $\geq 350 \mathrm{~ms}$ and Fridericia QTc of $\geq 340$ ms can be incorporated into clinical decision-making, but QTc prolongation at peak heart rates provides no additive diagnostic information in addition to ST depression. Furthermore, these simpler heart rate corrections of the QT interval at maximal exercise are not practical for routine clinical use. Further maximal exercise OTc studies which utilize rate correction factors or are estimated for each individual $\mathrm{OT} / \mathrm{RR}$ variability may be more beneficial in determining the additive diagnostic value of QTc at maximal exercise for the detection of $C A D$ presence and severity.

\section{References}

1. Crow RS, Hannan PJ, Folsom AR. Prognostic significance of corrected QT and corrected JT interval for incident coronary heart disease in a general population sample stratified by presence or absence of wide QRS complex: the ARIC Study with 13 years of follow-up. Circulation 2003;108:1985-9.

2. Rickards $A F$, Norman J. Relation between $\mathrm{OT}$ interval and heart rate. New design of physiologically adaptive cardiac pacemaker. Br Heart J 1981;45:56-61.

3. Davey P, Bateman J. Heart rate and catecholamine contribution to QT interval shortening on exercise. Clin Cardiol 1999;22:513-8.

4. Schijvenaars BJ, van Herpen G, Kors JA. Intraindividual variability in electrocardiograms. J Electrocardio/ 2008;41:190-6.

5. Sohaib SM, Papacosta O, Morris RW, Macfarlane PW, Whincup PH. Length of the OT interval: determinants and prognostic implications in a population-based prospective study of older men. J Electrocardiol 2008:41:704-10.

6. Bazett HC. An analysis of the time-relations of electrocardiograms. Heart 1920;7:353-70.

7. Fridericia LS. Die systolendauer im elektrokardiogramm bei normalen menschen und bei herzkranken. Acta Med Scan 1920;53:469-86.

8. Hodges M, Salerno D, Erlinen D. Bazett's QT correction reviewed-Evidence that a linear QT correctionfor heart is better. J Am Coll Cardiol 1983;12:694.

9. Sagie A, Larson MG, Goldberg RJ, Bengtson JR, Levy D. An improved method for adjusting the OT interval for heart rate (the Framingham Heart Study). Am J Cardiol 1992;70:797-801.

10. Rautaharju PM, Surawicz B, Gettes LS, et al. AHA/ACCF/HRS recommendations for the standardization and interpretation of the electrocardiogram: part IV: the ST segment, T and U waves, and the QT interval: a scientific statement from the American Heart Association Electrocardiography and Arrhythmias Committee, Council on Clinical Cardiology; the American College of Cardiology Foundation; and the Heart Rhythm Society. Endorsed by the International Society for Computerized Electrocardiology. J Am Coll Cardio/ 2009;53:982-91.

11. Miranda CP, Liu J, Kadar A, et al. Usefulness of exercise-induced STsegment depression in the inferior leads during exercise testing as a marker for coronary artery disease. Am J Cardiol 1992;69:303-7.

12. Takase $B$, Masaki $N$, Hattori $H$, Ishihara M, Kurita A. Usefulness of automatic QT dispersion measurement for detecting exercise-induced myocardial ischemia. Anadolu Kardiyol Derg 2009;9:189-95.

13. Ulgen MS, Karadede A, Alan S, Temamoğulari AV, Karabulut A, Toprak $\mathrm{N}$. Contribution of the peak exercise QT dispersion to the accuracy of an exercise test during the evaluation of coronary artery disease. Acta Cardiol 2000;55:335-9.

14. Chugh SS, Reinier K, Singh T, et al. Determinants of prolonged OT interval and their contribution to sudden death risk in coronary artery disease: the Oregon Sudden Unexpected Death Study. Circulation 2009; 119:663-70.

15. Goldenberg I, Moss AJ, Zareba W. OT interval: how to measure it and what is "normal". J Cardiovasc Electrophysio/ 2006;17:333-6.

16. Gensini GG. A more meaningful scoring system for determining the severity of coronary heart disease. Am J Cardiol 1983;51:606.

17. Gianrossi R, Detrano R, Mulvihill D, et al. Exercise-induced ST depression in the diagnosis of coronary artery disease. A meta-analysis. Circulation 1989;80:87-98.

18. Aytemir K, Maarouf N, Gallagher MM, Yap YG, Waktare JE, Malik M. Comparison of formulae for heart rate correction of QT interval in exercise electrocardiograms. Pacing Clin Electrophysiol 1999;22:1397401.

19. Luo S, Michler K, Johnston P, Macfarlane PW. A comparison of commonly used QT correction formulae: the effect of heart rate on the QTc of normal ECGs. J Electrocardio/ 2004;37 Suppl:81-90.

20. Rautaharju PM, Zhang ZM. Linearly scaled, rate-invariant normal limits for QT interval: eight decades of incorrect application of power functions. J Cardiovasc Electrophysio/ 2002;13:1211-8.

21. Chauhan VS, Krahn AD, Mitoff P, Klein GJ, Skanes AC, Yee R. Sudden intense exercise increases $\mathrm{QT}$ heart rate slope and T wave complexity in long OT syndrome and normal subjects. Pacing Clin Electrophysio/ 2004; 27:1415-23.

22. Kannankeril PJ, Goldberger JJ. Parasympathetic effects on cardiac electrophysiology during exercise and recovery. Am J Physiol Heart Circ Physiol 2002;282:H2091-8.

23. Mittleman MA, Siscovick DS. Physical exertion as a trigger of myocardial infarction and sudden cardiac death. Cardio/ Clin 1996;14:263-70.

24. Schwartz PJ, Priori SG, Spazzolini C, et al. Genotype-phenotype correlation in the long-OT syndrome: gene-specific triggers for life-threatening arrhythmias. Circulation 2001;103:89-95.

25. Hirao H, Shimizu W, Kurita T, et al. Frequency-dependent electrophysiologic properties of ventricular repolarization in patients with congenital long OT syndrome. J Am Coll Cardiol 1996;28:1269-77.

26. Shaw RM, Rudy Y. Electrophysiologic effects of acute myocardial ischemia: a theoretical study of altered cell excitability and action potential duration. Cardiovasc Res 1997;35:256-72.

27. Lauer MS, Pothier CE, Chernyak YB, et al. Exercise-induced QT/R-R-interval hysteresis as a predictor of myocardial ischemia. J Electrocardiol 2006;39:315-23.

28. Malik M. Problems of heart rate correction in assessment of drug-induced OT interval prolongation. J Cardiovasc Electrophysiol 2001;12: 


\section{KCI Korean Circulation Journal}

$411-20$.

29. Magnano AR, Holleran S, Ramakrishnan R, Reiffel JA, Bloomfield DM. Autonomic nervous system influences on $\mathrm{QT}$ interval in normal subjects. J Am Coll Cardio/ 2002;39:1820-6.
30. Arrowood JA, Kline J, Simpson PM, et al. Modulation of the OT interval: effects of graded exercise and reflex cardiovascular stimulation. J App/ Physiol (1985) 1993;75:2217-23. 